\title{
MISCELÁNEA
}

\section{QUAL CON ANTECEDENTE EN ESPAÑOL ANTIGUO}

\section{Preliminar}

El presente trabajo * pretende contribuir al conocimiento de la historia del relativo medieval qual / el qual a partir de los datos proporcionados por un conjunto de textos que se relacionan al final. Se intenta una ampliación de los datos empíricos de estudios previos (Gessner, 1894; Kuersteiner, 1911) y, al mismo tiempo, una ordenación de los hechos que permita hallar un hilo conductor entre usos aparentemente inconexos. $\mathrm{El}$ procedimiento seguido ha sido el de analizar estos usos tomando como punto de referencia el desenvolvimiento de la antigua correlación tal... qual..., que parece regular las diferentes configuraciones adoptadas por el relativo medieval.

\section{UsOS CORRELATIVOS}

El español qual y el latino qui coinciden en haber funcionado, antes de ser relativos, como miembros de una correlación cuyos dos elementos

* Las páginas que siguen constituyen una versión corregida y muy resumida del capítulo correspondiente de $\mathrm{mi}$ tesis doctoral sobre Los pronombres relativo-interrogativos en español medieval, leída en la Univ. Autónoma de Madrid en julio de 1984 y juzgada por los profesores D. Diego Catalán, D. Gregorio Salvador, D. Francisco Marcos Marín (ponente), D. Emilio Ridruejo y D. Ignacio Bosque. Obtuvo la calificación de sobresaliente cum laude.

LXV, 3.0 - 4.0-9 
* to $\mathrm{y}{ }^{*} q u^{\circ}$, tal y qual fueron originariamente un anafórico y un interrogativo indefinido (Leumann-Hoffmann, 1965, núm. 298) ${ }^{1}$. Nada nos autoriza en principio a afirmar que el proceso que ha llevado a constituir el relativo qui latino a partir de una antigua correlación tenga que repetirse con las mismas etapas en el caso de qual. Ello no obsta, sin embargo, para que señalemos una serie de significativas coincidencias observables en la historia de ambos pronombres.

Haudry (1973 y 1979) ha querido poner el origen de la subordinación latina en relación con un proceso de inversión de la antigua correlación * $q u^{\circ} \ldots{ }^{*}$ to..- y ulterior eliminación del correlativo anafórico:

1) qui homo... to...

2) to homo... qui...

3) (is) homo... qui...

Cae fuera de nuestros objetivos la tarea de verificar la hipótesis de Haudry en lo que al latín se refiere. Pero sí nos vemos obligados a observar que los datos relativos a qual que nuestros textos proporcionan permiten una ordenación similar a la efectuada por Haudry para los hechos latinos.

En efecto, hemos documentado la estructura qual... tal..., que llamaremos tipo I, en la que nunca aparece involucrado un sustantivo. En esta estructura qual tiene siempre su valor cualitativo originario:

demas el homne debe asmar e comedir / que qual aqui fiziere tal habra de padir (Apol: 413cd)

«mudase el amor con el nueuo entendedor» e qual faze al primero, tal al segundo e tal al tercero (Troy-360: 1-3)

quales dios les mostrase faser signos con la mano / que tales los fesiese e fueles consejo sano (LBA-G: 51cd)

bien o mal, qual puntares, tal te dira çierta mente (ibid-S: 70b)

qval la ellos ouieron a uos tal la dexaron (Alex-0: 207d).

La segunda estructura correlativa que encontramos, a la que llamaremos tipo I', es similar a la anterior en lo que al orden de elementos se refiere, pero aparece aquí un sustantivo en el primer miembro de la correlación. Qual funciona como adjunto de este sustantivo. En este tipo, cuya fórmula general es qual cosa... tal..., el valor cualitativo originario se encuentra muy desdibujado o casi totalmente oscurecido:

1 Se discute, sin embargo, si el tema * qu० era interrogativo antes que indefinido, o viceversa. La cuestión es, para nuestros fines, irrelevante (cf. Spitzer, 1941; Fowler, 1931; Szemerényi, 1978, págs. 268-269, y la bibliografía allí citada). 
qual senior uoluerit, tale se prendant (FMadrid-LXVIII: 18)

a qual sennor serviestes recibredes tal dado (Signos: 32d)

ca en qual iuizio iudgaredes, en tal seredes iudgados, e qual medida midieredes, con tal midran a uos (SMateo-7: 1-2)

qual pleito tu quisieres, nos tal te lo fagamos (Apol-89c).

Llamaremos tipo II a la estructura configurada según la fórmula tal... qual..., sin la presencia de un sustantivo. En esta estructura tal y qual suelen conservar su valor cualitativo originario:

Fazer telo he dezir que tal eres qual digo yo (Cid: 3389)

En todas guisas tales sodes quales digo yo (ibid: 3454 ).

Por fin, la estructura de tipo II' es aquella que, similar a la anterior, contiene un sustantivo en el primer miembro de la correlación (tal cosa... qual...). En algunos casos el valor cualitativo de los dos elementos se percibe todavía con nitidez:

tal pobre qual tu veyes, avez so escapado (Apol: 129d)

si mas ante mi vienes, recibras tal amor / qual fezist a Tarsiana e non otro mejor (ibid: $389 \mathrm{~cd}$ ) 2 .

Pero en la mayoría de los casos dicho valor es, cuando menos, muy borroso; tal está cerca de convertirse en un relativo puro y qual es casi un relativo:

non podien de gran cueyta nin leer nin orar / ca perdien tal consejo qual non podrien trobar (SMillán: 297cd)

E non vos semeje desaguisada cosa de Colcas auer muchas de tales donas quales auedes oydas (Troy-359: 5-6)

que fiz fasta aqui seruiçio / en tal lugar qual deuia (ibid-X: 127-128)

jamad, dueñas, amalde tal omne qual debuxo! (LBA S: 1490b).

El segundo proceso postulado por Haudry es la elisión del antecedente. Estudiaremos a continuación la repercusión de dicha eliminación en las cuatro estructuras anteriores.

La eliminación de tal en la estructura de tipo I (qual... tal...) supone la sustitución de éste por un elemento pronominal que refiere anafóricamente a qual ${ }^{3}$ :

\footnotetext{
2 En algún caso se produce la atracción de los dos elementos: «bien atal qual sea, dime toda su fechurax (LBA-S: 1484c).

3 Del mismo modo, en latín, cuando la subordinada introducida por qui antecede a la principal, el antecedente es reproducido en ésta. Bassols (1971, vol. II, pág. 238) cita el siguiente caso: ain tabellis quos consignaui heri latrones, ibus dinumerem stipendium* (Plauto).
} 
que qual elli mandasse, ellos essi querrien (Loor: 140d)

En qual vinyere el fuego del cielo, esse sea el Sennor (Faz: 122)

qual de todos estos a mas dignidades e mas firmas en la figura, aquel sera el significador de la cogitación (LConp-I, 24a: 32-35).

La supresión de tal en la estructura de tipo II da lugar a construcciones de qual relativo sin antecedentes:

que escogiesse destas iii cosas qual queria mas (Faz: 142)

dixieron que mandasse quales quisiese matar (LBA-S: 83c)

Otro dia de mañana metense a andar / a qual dizen Medina ivan albergar (Cid: 2878-2879).

La supresión de tal en la estructura I' da lugar, como en las construcciones latinas con qui antepuesto, a la aparición de un elemento pronominal o adverbial que señala a qual en el segundo miembro de la correlación:

e en qual parte ouiere mayor dignidat entre el ascendente e la VII casa, a aquella parte se tira e ama mas (LConp-II, 92d: 22-25)

$E$ en qual casa entraredes, alli aluergat (SLucas-9:4)

e por qual part uieren que fizieren menos danno, por y den carrera (FBéjar: 56).

La estructura anterior (qual cosa... pron/adv) ha podido perder el elemento pronominal o adverbial que sustituye a tal en los casos en que ambos correlatos desempeñan la misma función ${ }^{4}$ :

Con qual abito pudo pensose de mover (SMillán: 77a)

de qual muerte tu mueres me faga Dios morir (Alex-P: 1785d).

La ausencia de antecedente de cualquier tipo ha permitido reordenar la frase, haciendo que la relativa que se ha formado ocupe el segundo lugar en la estructura subordinante:

te prometo qual cosa tu quisieres (Apol: 40d) [en vez de: qual cosa tu quisieres (esa) te prometo]

diole qual cena pudo (ibid: 139c) [en vez de: qual cena pudo (esa) diole].

Qual es aquí una suerte de adjetivo relativo especificativo cuyo antecedente va incluido en la misma subordinada ${ }^{5}$.

4 Lo mismo ocurría en latín con el pronombre qui. Bassols (ibid., pág. 237) afirma que este relativo perdía su antecedente acuando éste deberia ser el pronombre anafórico, especialmente cuando el antecedente y el relativo están en el mismo caso o ambos están en casos rectos.

5 Conviene observar que, en lo que al latín se refiere, las gramáticas no suelen reconocer el tipo qui homo como una estructura relativa independiente y diferente 
Finalmente, la eliminación de tal en II' (tal cosa... qual...) nos proporciona una estructura subordinada relativa apositiva en la que el sustantivo anteriormente modificado por tal funciona como antecedente; qual funciona como un especificador que, retroactivamente, delimita la referencia del antecedente ${ }^{6}$ y equivale a el que (Lapesa, 1975, pág. X, número 3 y nota 5):

esto es, que ayades lit qual uiestes en mi (Fil-I: 30)

Rica muger e fiia de vn porqueriso vil / escogera marido qual quesiese dellos mill (LBA-G: 600ab)

Era costumbre que el dia de la Pascua daua el merino al pueblo un preso, qual ellos quisiessen (SMateo-27: 15)

enbio llamar otros dos grandes sabios, quales el nombro (DozeSabiosLXVI: 117)

Cavalgo Minaya con Per Vermudoz / e Martin Antolinez, el Burgales de pro, / con dozientos cavalleros quales mio Cid mando (Cid: 2836-2838).

Si la ordenación de los datos que proponemos es correcta, tenemos buenas razones para pensar que en la base de la doble relación existente entre qual y su antecedente en español medieval (doble relación que afecta al orden de los elementos y a la referencia del sustantivo) se encuentra la convivencia en español antiguo de dos tipos básicos e inversos de correlación. En las dos configuraciones que adopta la correlación de qual con tal están previstas las posibles relaciones que qual mantendrá con respecto al sustantivo una vez que la supresión de tal se haya producido.

\section{QUAL ANTECEDENTE DEL RELATIVO}

Resulta oportuno este momento para mencionar un tipo peculiar de estructura relativa en la que qual con sustantivo o sin él funciona como

de homo qui. Así, Leumann-Hofmann (1965, núm. 304) y Bassols (ibid., núm. 231) incluyen las construcciones del primer tipo en apartados dedicados a lo que llaman atracción del antecedente en la subordinada relativa, entendiendo, pues, que la estructura qui homo es derivada de la estructura homo qui, por medio del citado proceso de atracción. Rubio (1982) protesta contra esta interpretación tan generalizada y se apoya en una comparación con los relativos españoles para defender el carácter autónomo del tipo qui homo. Dice literalmente (pág. 287): «Nosotros vemos como construcción normal la siguiente: Cuanto dinero ganaba lo daba a los pobres, y nadie piensa aquí en una desviación a partir de todo el dinero que ganaba lo daba a los pobres. De la misma manera han de verse como normales las construcciones adjetivo-relativas..

6 Más ejemplos en Gessner (ibid., pág. 453) y Keniston (1937, núm. 15.82). 
antecedente del relativo que, obteniéndose de esta forma una estructura aparentemente redundante qual (cosa) que ${ }^{7}$ :

Fraire ho seglar, qual que hi for, aya el castiello por heredades en que lauren (DLE-327: 20-21)

Equal que vaya contra ello sea maldicto de Dios (ibid-66: 25)

gano el moleo de Oiouarth del molino de suso del abat don P. qual hora que fuesse a la que moliesse (ibid-80: $3-4$ )

a qual parte que iba tenieli las fronteras (SMillán: 53b)

Estos son iudizios que comendo delant ellos por qual cosa que abiniesse (Faz: 77)

A quales $\mathrm{V}$ peones que aduxiere cada nouena catenlos fidelmientre el iudez con los alcaldes (FBéjar: 695).

Pensamos que para comprender estos usos es importante no perder de vista la existencia de dos tipos diferentes de correlación de cualidad, con las correspondientes estructuras relativas derivadas, y, sobre todo, hacer notar que dicha convivencia introduce una dualidad en el comportamiento sintáctico de qual que pudo favorecer la aparición de estas construcciones.

Examinemos nuevamente el que hemos llamado tipo I' (qual cosa... tal...). Los dos miembros que constituyen esta correlación parecen tener idéntico peso sintáctico y semántico: qual, como adjunto de un sustantivo, establece una cualidad o mención indefinida que es reproducida en el segundo miembro por tal:

a qual sennor servieron, ovieron tal soldada (Loores 138-d) (=sirvieron a un señor de una cierta clase: tuvieron una soldada de esa misma clase) en qual casa entraredes, elli aluergat (SLucas-9: 14) (=entraréis en alquna casa: albergad en esa casa).

Pensamos que en frases como las anteriores qual es todavía un indefinido ${ }^{8}$ y tal un anafórico.

7 En Moignet (1973, págs. 49 y 116) encontramos citas similares del francés antiguo.

8 Esta idea aparece sugerida pero no enunciada en el clásico trabajo de Kroll (1912). Por lo demás, la relación entre correlación y subordinación viene sugerida también en el siguiente párrafo de Meillet (1966), escrito con otro propósito: «Este sistema de is... qui... y de tam... quam..., tum... cum... es importante para la articulación de las frases. Permite la subordinación de las frases relativas a las frases principales de una manera muy sensible y con solidez. Gracias, en parte, a este sistema (...), el latín dispone de la subordinación expresiva y sólidamente establecida que le da un carácter original y una fuerza singular para expresar un pensamiento bien analizado y bien conjuntado» (pág. 101). 
En la correlación II' (tal cosa... qual...) el determinante tal establece una referencia definida; qual funciona ya como un anafórico que especifica esa referencia:

recibras tal amor / qual fezist (Apol: 389cd) (=recibirás ese [tipo de] amor: el que hiciste).

A nuestro juicio, el elemento qual que aparece en los grupos citados establece una mención indefinida (con eventual valor cualitativo) según lo previsto en los tipos I y I', pero, fuera de la configuración correlativa, actúa como puede hacerlo cualquier otro indefinido, constituyendo una frase nominal que puede funcionar como antecedente del relativo 9.

\section{EL QUAL}

El relativo el qual aparece documentado en nuestro material con abundancia suficiente para los fines que nos proponemos. Son éstos básicamente dos: averiguar sus propiedades específicas (en particular en comparación con las del simple qual) y, por otro lado, encontrar una posible justificación a la aparición del artículo, habida cuenta que la suposición de un hipotético origen * ille qualis no parece sostenible (Lapesa, 1975, núm. $X$, nota 3 ).

Se ha afirmado que el qual es un pronombre esencialmente explicativo (Gessner, pág. 453), lo que parece ser cierto en la época que estudiamos, puesto que, salvo alguna excepción:

demostrolis la forma con la qual bapteassen (Loores: 131b)

el qual en nuestros textos es siempre explicativo ${ }^{10}$ :

disso, "Agora veo de plan la medezina / la qual me dara sana con la gracia divina” (SMillán: 149cd)

\footnotetext{
9 En un trabajo reciente, Rivero (1984) se enfrenta desde una metodologia transformacional muy elaborada al análisis de lo que esta autora considera también una doble sintaxis de los relativos medievales. Sus conclusiones, más allá incluso de lo propiamente lingüístico tienen relevancia en el campo de la teoría de la adquisición del lenguaje. No se explora, sin embargo, la eventual relación entre usos relativos y correlativos de los pronombres, punto éste en el que haremos hincapié en este trabajo.

10 También en el siglo Xvi parece ser el qual casi siempre explicativo, a juzgar por los datos de Keniston (ibid., núm. 15.122). El nacimiento del francés lequel se ubica igualmente en la Edad Media, también con valor explicativo (Moignet, 1973, páginas 165-166).
} 
castigando a los apostolos por el Espiritu Sancto, a los quales el se mostro uiuo... (Hechos-1: 2-3)

... quando el omne toma martitrio por la fe, la qual Dios quiera que hayan aquellos que la desean (LCE-XLVI: 58-60)

e assi encargados sodes uos, ante los oios de los quales Christo esta escripto (Gál-3:1).

En ocasiones el qual hace referencia, no a un elemento de la frase anterior, sino a la idea contenida en ella:

Que muchos fablan al señor a su voluntad por le conplazer e lisonjar, negandole la verdad, lo qual es manifiesto yerro (DozeSabios-XIV: 12-13).

Usos como éste se sitúan, a nuestro juicio, en el umbral entre la referencia anafórica relativo-explicativa y la función anafórica propiamente demostrativa. El relativo el qual puede traspasar este umbral. Por lo pronto, como otros demostrativos, el qual proporciona, no una información complementaria de lo afirmado en la principal, sino que introduce una «nueva peripecia» (Fernández Ramírez, 1953, núm. 167, página 343) con valor semántico independiente. Ello le permite introducir un período sintáctico también independiente, en ocasiones tras punto ortográfico, como en el siguiente caso:

que nos escoio pora salut e a santiguamiento de Espirito e en fe de uerdat. En la qual uos llamo por la nuestra prigación (2-Tes-2: 12-13).

El qual, igual que los demostrativos, puede efectuar una referencia a un elemento del campo sintáctico que puede ser reproducido en la nueva oración ". Obsérvese, por ejemplo, el comportamiento del demostrativo en la frase siguiente, citada por Fernández Ramírez:

hemos labrado una choza con cuatro palitroques y un poco de paja. Esta choza es para nosotros un lugar sagrado.

El qual se comporta en las frases siguientes como el demostrativo de la anterior:

era so cormano e so esposo de Sancta Maria, de la qual Sancta Maria fo nado Ihesus (LRegum-7: 2-3)

E agora esto puesto en iuizio por la esperança del prometimiento que fue fecho de Dios a nuestros padres...; de la qual esperança so acusado de los iudios (Hechos-26: 6-7)

11 Lo mismo ocurría con qui en latín arcaico, especialmente en textos arcaicos y, a veces, en César. Cf. Bassols (ibid., núm. 233, pág. 241) que cita: «erant omnino duo itinera, quibus itineribus domo exire possent" (César). 
Assi Christo no se escogio el mismo pora seer obispo... El qual Christo, en los dias de su carne, priezes e ruegos fizo a Dios (Heb.: 5-7).

Es propia también de los demostrativos la que Fernández Ramírez (núm. 129, pág. 250) llama anáfora difusa «en la que el pronombre adjetivo va con un sustantivo que no se repite, sino que es el resultado de una elaboración conceptual, mediante la cual se interpreta una palabra o el sentido de un grupo de palabras ya pronunciado"; Fernández Ramírez cita el siguiente ejemplo:

No he bailado porque a Julianita nadie le decía nada, y como se muere de envidia, no he querido darle ese mal rato.

Los siguientes usos de el qual nos parecen similares ${ }^{12}$ :

Estas cosas sennalaua el Santo Espirito, non seyendo aun descubierta la carrera de los santos, estando aun la primera tienda en su estado.

La qual semeiança es deste tiempo dagora (Heb-9: 8-9)

e gozo me ementando la fe que es en ti no enfinta, la que fue primero en tu auuela Loyda e en tu madre Eunica, e so cierto que otrossi es en ti; por la qual cosa te amonesto que despiertes la gracia que es en ti (2-Tim-1: 5)

E assi, hermanos, non somos fiios de sierua, mas de libre, en la qual libertad Christo nos fizo libres (Gál.-4:31).

\section{CONCLUSIÓN}

Los datos recogidos y analizados en este trabajo aportan, a nuestro juicio, indicios suficientes para arriesgar una hipótesis sobre el origen del relativo el qual. En efecto, ¿qué tipo de procesos han podido convertir el adverbio latino qualis en un relativo explicativo precedido de artículo? No hay, desde luego, datos empíricos latinos o romances que apoyen la idea de que el grupo el qual nació con el (o ille) como antecedente. Parece, por el contrario, que el nacimiento de este relativo compuesto debe ponerse en relación con otros procesos evolutivos experimentados por qual. En particular, la existencia de dos tipos inversos de correlación con tal han podido configurar los usos de qual que tras la pérdida del antecedente podemos llamar relativo. Recuérdese que uno de estos usos (previsto por la correlación de tipo II') ha dado lugar a

12 Bassols (ibid., pág. 241) cita una construcción parecida, en la que está presente qui: $\alpha . .$. in aedem Telluris ...in quo templo...» (Cicerón). 
un relativo apositivo que funciona como especificador de la referencia del antecedente.

La estructura apositiva que de esta forma ha surgido ofrece a la lengua medieval las bases para la creación de un nuevo uso de qual en el que, conservando el carácter apositivo de las construcciones anteriores, funcione, no para delimitar la referencia del sustantivo antecedente, sino para introducir aposiciones de carácter explicativo. La aparición del artículo podría entonces justificarse por alguna de las dos razones siguientes, no incompatibles:

a) Como demostrativo (o relativo-demostrativo), el qual puede señalar gracias al artículo la concordancia de género con el sustantivo antecedente, concordancia que el sistema qual no proporciona.

b) Por medio del artículo, la lengua antigua ha podido distinguir dos usos apositivos del relativo qual claramente diferentes: el uso especificador de qual ya comentado y el uso explicativo de el qual, también apositivo, que no aporta información sobre la referencia del antecedente, sino que proporciona un contenido adicional a lo expresado en la frase principal.

JAVIER ElVIRA

\section{NOMINA DE TEXTOS}

Documentos lingüísticos de España (Reino de Castilla). Ed. de R. Menéndez Pidal; Anejo DXXXIV de la RFE, Madrid, 1966 (Abrev. DLE).

Fuero de Madrid. Ed. de Rafael Lapesa; Ayuntamiento de Madrid, Madrid, 1962 (Abrev. FMadrid).

Liber Regum. Ed. de Louis Cooper; Zaragoza, Institución «Fernando el Católico», 1970 (Abrev. LRegum).

Fazienda de Ultramar. Ed. de Moshe Lazar, Salamanca, "Acta Salmanticensia», 1965 (Abrev. Faz).

Gonzalo de Berceo: Vida de San Millán de la Cogolla. Ed. de Brian Dutton; Londres, Thamesis Books, 1967 (Abrev. SMillán).

- El duelo de la Virgen, Los himnos, Los loores de Nuestra Señora, Los signos del juicio final. Ed. de Brian Dutton, Londres, Thamesis Books, 1975 (Abrev. Duelo, Himnos, Loores, Signos).

El Evangelio de San Mateo. Ed. de Th. Montgomery; Anejo VII del B.R.A.E.; Madrid, 1962 (Abrev. SMateo).

El Nuevo Testamento. Ed. de Th. Montgomery y S. Baldwin; Anejo XXII del B.R.A.E., Madrid, 1970 (Abrev. Fil.-Filipenses; Gál. Gálatas; Heb. Hebreos; Hechos. Hechos de los Apóstoles; SLucas. Ev. de San Lucas; Tes. Tesalonicenses. Tim. Timoteo). 
Libro conplido en los iudizios de las estrellas. Ed. de G. Hilty; Madrid, R.A.E., 1964 (Abrev. LConp).

Fuero de Bejar. Ed. de Juan Gutiérrez Cuadrado; Salamanca, «Acta Salmanticensia», 1974 (Abrev. FBéjar).

Libro de Alexandre. Ed. de R. S. Willis; Princeton, Elliot Monographs, 1934 (Abrev. Alex-O o -P).

Cantar de Mio Cid. Ed. de R. Menéndez Pidal, 4.a ed., Madrid, Espasa-Calpe, 1969 (Abrev. Cid).

Historia troyana en prosa y verso. Ed. de R. Menéndez Pidal, en "Textos Medievales Españoles", págs. 179-419; Madrid, Espasa-Calpe, 1976 (Abrev. Troy).

Arcipreste de Hita: Libro de buen amor. Ed. de M. Criado de Val; Madrid, CSIC, 1972 (Abrev. LBA-S o G).

Libro de Apolonio. Ed. de Manuel Alvar; Fund. Juan March-Editorial Castalia, Madrid, 1976 (Abrev. Apol).

El libro de los doze sabios (Tratado de la nobleza y lealtad). Ed. de K. Walsh; Madrid, Anejo XXIX del B.R.A.E., 1975 (Abrev. DozeSabios).

Don Juan Manuel: Libro del cauallero et del escudero. Ed. de J. M. Blecua; en Obras Completas, vol. I, págs. 39-116, Madrid, Gredos, 1983 (Abrev. LCE).

\section{REFERENCIAS}

Alvar, M., y B. Pottier: Morfología histórica del español, Madrid, Gredos, 1983.

Bassols de Climent, M.: Sintaxis Latina, 2 vols., C.S.I.C., Madrid, 1971.

Bello, A (y Cuervo, R. J.): Gramática de la lengua castellana, 7.' ed., Buenos Aires, Sopena, 1964.

Fernández Ramírez, Salvador: Gramática española. Los sonidos, el nombre y el pronombre, Madrid, Rev. de Occidente, 1951.

Fowler, F. H.: «The origin of the latin qui-clauses", Language, 7, 1931, págs. 14-29.

Gessner, E.: «Das spanische Relativ- und Interrogativpronomen», ZRPH, XVIII, 1894, págs. 449-497.

Haudry, Jean: «Parataxe, hypotaxe et corrélation dans la phrase latine», Bull. de la Soc. de Ling. de Paris, 68, 1973, págs. 147-186.

- «L'anteposition de la relative en Indo-Européen», La Linguistique, 15, I, 1979, páginas 101-110.

Keniston, H.: The Syntax of Castilian Prose, Chicago, 1937.

Kuersteiner, Albert F.: "The use of the relative pronoun in the Rimado de Palacio», Revue Hispanique, 24, 1911, págs. 46-70.

Kroll, W.: «Der lateinische Relativesatz», Glotta, 3, 1912, págs. 1-18.

Lapesa, R.: «El artículo como antecedente del relativo», incluido en F. Marcos: Aproximación a la Gramática española, Madrid, Cincel, 1975, págs. IX-XVII.

Leumann, M., y J. B. Hofmann, J. B.: Lateinische Grammatik (con apéndice de Szantyr), 1965.

Meyer-Lübke, W.: Grammaire des langues romanes, 3 vols., París, 1900.

Moignet, Gerard: Grammaire de l'Ancien Français. Morphologie-Syntaxe, Paris, Klincsieck, 1973. 
Meillet, A.: Esquisse d'une Histoire de la Langue Latine (utilizamos trad. esp. en Avesta, Reus, 1980), 1966.

Rivero, M. L.: «Diachronic syntax and learnability. Free relatives in 13th century Spanish», Journal of Linguistics, 20, 1984, págs. 80-131.

Rubio, L.: Sintaxis estructural del latín, Barcelona, Ariel, 1982.

Spitzer, L.: «Interrogativo e indefinido», $R F H$, III, 1941, págs. 1-8.

Szemerényi, O.: Introducción a la lingüistica comparativa, Madrid, Gredos, 1978. 